\title{
Aborto Habitual. Tratamiento con Gonadotropina Coriónica Humana
}

\section{INTRODUCCION}

El aborto espontáneo es la complicación más frecuente del embarazo, ocupando el primer lugar entre las causas de fracaso de la gestación. Se trata entonces de un problema sanitario de gran importancia, pues el número de vidas en potencia que se pierden es aproximadamente igual al número de adultos que poseen enfermedades cardiovasculares.

La frecuencia del aborto espontáneo fluctúa entre el 1.0 y el $18 \%$ aproximadamente $(1,2,3)$. Dentro de este grupo de mujeres existen aquellas que abortan espontáneamente 30 más embarazos consecutivos; son las Ilamadas abortadoras habituales que constituyen el $0.4 \%$ de todas las gestaciones y en las cuales las probabilidades de fracaso en un siguiente embarazo son mayores del $47 \%$ (4).

El presente trabajo sobre aborto habitual se dirigió fundamentalmente hacia aquellas mujeres en las cuales existe una inadecuada fase luteínica como causa principal en la pérdida de su gestación. Esta deficiente fase luteínica conduce a una disminución en la secreción de progesterona, la cual va a producir la pérdida del producto de la concepción.

* Sección de Fisiología de la Reproducción. Departamento de Obstetricia y Ginecología. División de Salud. Universidad del Valle. Cali. Colombia.
Diferentes autores $(5,6,7)$ han intentado corregir esta deficiencia de progesterona endógena adminitrando progesterona o progestinas exógenas con resultados negativos. Asi, Shearman y Garret (5) realizando un estudio doble ciego con caproato de hidroxiprogesterona encontraron que este fármaco no produjo incremento significativo en la excreción urinaria de pregnandiol en pacientes con 2 o más abortos espontáneos consecutivos. Goldzieher (6) encontró resultados similares administrando acetato de medroxiprogesterona por vía oral.

Un año más tarde, Klopper y Macnaughton (7) no encontraron ninguin beneficio con el uso de progesterona en la prevención del aborto en pacientes abortadoras habituales.

Por otra parte, trabajos más recientes de Strotty y col. (8), demostraron que cuando la hormona gonadotropina coriónica (HCG) se administra en la fase luteínica del ciclo menstrual, se obtiene un incremento significativo en los niveles plasmáticos de progesterona y 17 hidroxi progesterona. En cambio, administrada la HCG en ausencia de cuerpo lúteo, no se observa ningún cambio en los niveles sanguíneos de las hormonas mencionadas.

Sin embargo, el papel de la HCG como estimulante de la esteroidogénesis por el cuerpo lúteo después de la fertilización, no ha sido aun bien establecido. Basado en lo anterior, se diseñó la presente investigación cuyo objetivo fue el 
de tratar de demostrar un efecto estimulante de la HCG sobre la producción endógena de progesterona por el cuerpo lúteo del embarazo. Para ello se administró dosis repetidas de gonadotropina coriónica humana a pacientes embarazadas con antecedente de aborto habitual por insuficiencia luteínica.

\section{Pacientes y métodos}

Se seleccionaron 26 mujeres voluntarias en la Consulta Ginecológica del Hospital Universitario durante los años 1974 a 1978. De este grupo la mitad de ellas eran pacientes abortadoras habituales que deseaban ser estudiadas antes de iniciar una nueva gestación (tabla 1). Los criterios seguidos para la selección de estas pacientes eran que los siguientes exámenes estuviesen dentro de lo normal: Histerosalpingografía, glicemia pre y post-prandial, reacción para toxoplasmosis, cariotipo de la pareja, serología y urocitograma. Si los anteriores exámenes eran informados como normales se aconsejaba a la paciente un nuevo embarazo y regresar cuando tuviera una semana de falla menstrual. Al consultar nuevamente la paciente, se diagnosticaba embarazo en lo posible por métodos inmunológicos. Posteriormente se extraía sangre venosa antecubital para análisis de progestinas plasmáticas entre 5 a 9,10 a 14 y 15 a 19 semanas de gestación. Inmediatamente se obtenía la primera mues- tra de sangre, se iniciaba la terapia hormonal administrando Gonadotropina coriónica a dosis de 5.000 U. I. por V. I. M., cada tercer día hasta el séptimo mes de la gestación. A partir de esta edad de embarazo se suspende la administración del fármaco y se dejaba evolucionar espontáneamente.

El segundo grupo (control) estuvo constituido por 13 embarazadas normales y a quienes se les hizo determinación de progestinas plasmáticas entre las 5 a 9,10 a 14 y 15 a 19 semanas de gestación. No recibieron ningún tipo de medicación hormonal y el embarazo transcurrió sin patología detectable.

Las progestiras plasmáticas se cuantificaron mediante una técnica de unión protéica competitiva, descrita por Johansson (9) y modificada parcialmente por nuestro laboratorio. Todas las pacientes estudiadas se dividieron finalmente en 3 grupos de acuerdo con la edad gestacional en que se obtuvo la muestra de progestinas. Se calculó el promedio y el error estándar de la medida. Se utilizó la prueba de " $t$ " para muestras independientes, para verificar la significación estadística de los resultados.

Se comparó también el peso de los recién nacidos expresado en gramos mediante una prueba de " $t$ " para muestras independientes. El sexo de los neonatos y la vía de parto (cesárea o aborto va-

TABLA 1

NUMERO DE ABORTOS ESPONTANEOS PREVIOS EN CADA UNA
DE LAS PACIENTES ESTUDIADAS

\begin{tabular}{|c|c|c|c|c|c|c|c|c|c|c|c|c|c|}
\hline Paciente & 1 & 2 & 3 & 4 & 5 & 6 & 7 & 8 & 9 & 10 & 11 & 12 & 13 \\
\hline Abortos previos & 3 & 6 & 9 & 3 & 4 & 3 & 4 & 5 & 4 & 3 & 3 & 4 & 7 \\
\hline
\end{tabular}


ginal) se compararon utilizando la prueba de Chi cuadrado. También se registró el puntaje de Apgar al $5^{\circ}$ minuto de vida.

\section{RESULTADOS}

Efectos de la gonadotropina coriónica sobre los niveles de progestinas

Entre 5 y 9 semanas de gestación, las pacientes embarazadas normales (E. N.) tuvieron un valor promedio de progestinas plasmáticas de $30.3 \pm 3.0$ ng./cc., mientras que en las embarazadas abortadoras habituales (A. H.) este valor fue de $10.7 \pm 0.9 \mathrm{ng} . / \mathrm{cc}$. La diferencia estadísticamente es significativa: $t=6,13, p<0.001$ (tablas 2 y 3 , fig. 1).

Entre 15 y 19 semanas de gestación, las progestinas plasmáticas fueron de $50.8 \pm 3.3 \mathrm{ng} . / \mathrm{cc}$., en las embarazadas normales y de $50.2 \pm 3.4 \mathrm{ng} . / \mathrm{cc}$. en las abortadoras habituales. Las diferencias

TABLA 2

VALORES DE PROGESTINAS EN EMBARAZADAS NORMALES

SEMANAS DE GESTACION

\begin{tabular}{lrrr}
\hline & 5 a 9 & 10 a 14 & 15 a 19 \\
\hline & 20.9 & 45.6 & 41.8 \\
& 30.2 & 42.6 & 58.2 \\
& 19.4 & 32.0 & 44.8 \\
& 43.4 & 34.0 & 37.0 \\
& 32.1 & 44.7 & 73.1 \\
$\mathrm{ng}$ & 26.9 & 16.0 & 32.0 \\
$\mathrm{CC}$ & 17.1 & 36.6 & 50.3 \\
& 28.9 & 37.7 & 40.1 \\
& 24.1 & 51.8 & 45.4 \\
& 58.9 & 56.5 & 60.2 \\
& 34.5 & 54.8 & 62.3 \\
\hline X & 29.7 & 47.0 & 63.1 \\
D. S. & 28.0 & 32.0 & 53.0 \\
E. S. & 30.3 & 40.8 & 50.8 \\
\hline
\end{tabular}

entre los 2 grupos ya no son estadísticamente significativas: $t=0.13, p>0.05$ (tablas 2 y 3 , fig. 1).

TABLA 3

VALORES DE PROGESTINAS EN ABORTADORAS HABITUALES

SEMANAS DE GESTACION

\begin{tabular}{lrrr}
\hline & 5 a 9 & 10 a 14 & 15 a 19 \\
\hline & 10.8 & 16.4 & 30.1 \\
& 11.3 & 15.1 & 38.9 \\
& 12.5 & 16.7 & 70.2 \\
& 7.3 & 21.4 & 70.3 \\
& 9.9 & 24.1 & 40.5 \\
ng & 14.0 & 21.3 & 48.5 \\
cc & 14.4 & 22.6 & 53.7 \\
& 9.1 & 20.2 & 60.0 \\
& 3.9 & 15.5 & 51.8 \\
& 8.0 & 19.7 & 49.5 \\
& 10.0 & 18.8 & 41.2 \\
& 16.0 & 21.9 & 40.8 \\
\hline X & 13.0 & 21.0 & 57.1 \\
D. S. & 10.7 & 19.6 & 50.2 \\
E. S. & 3.3 & 3.2 & 12.1 \\
\hline & 0.9 & 0.9 & 3.4 \\
\hline
\end{tabular}

\section{Peso de los recién nacidos}

El promedio de peso al nacimiento de los neonatos hijos de las $E$. N. fue de $3.392 .0 \pm 86.8 \mathrm{~g}$, mientras que los hijos de las A. H. tuvieron un peso promedio de $3.770 .0 \pm 90.0 \mathrm{~g}$, diferencia significativamente estadística: $t=296$, $\mathrm{p}<0.01$ (fig. 2).

\section{Sexo de los recién nacidos}

La proporción de neonatos de sexo masculino y femenino (5 y 8 respectivamente) en el grupo de E. N., no fue significativamente diferente de la del grupo de A. H. (4 masculinos, 5 femeninos): Chi cuadrado $=0.43, p>0.05$ (fig. 3). 


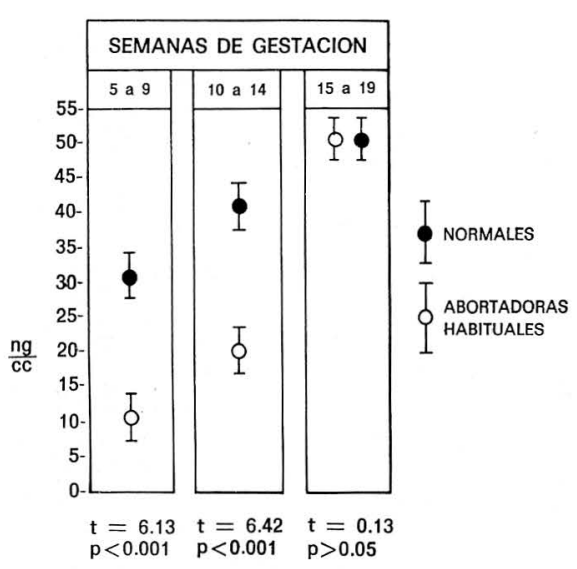

Fig. 1. - Niveles de progestinas plasmáticas en embarazadas normales y en mujeres con antecedentes de aborto habitual. Entre 5 a 9 y 10 a 14 semanas de gestación, las diferencias son estadísticamente significativas. Entre 15 a 19 semanas, después de recibir solamente gonadotrofina coriónica humana, los valores de progestinas plasmáticas son sensiblemente iguales en ambos grupos.

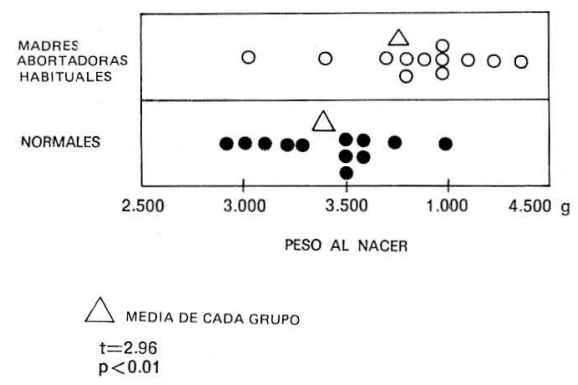

Fig. 2 - Distribución del peso al nacimiento (en g) de cada neonato en ambos grupos. En promedio, los hijos de pacientes con aborto habitual previo pesan $380 \mathrm{~g}$ más que los hijos de embarazadas normales.

\section{Método de terminación del embarazo}

La proporción de terminación quirúrgica del embarazo fue mayor en el grupo de pacientes A. H. (4 cesáreas, 9 partos vaginales) que en el grupo de las $E$. N. donde solamente hubo 2 cesáreas por 11 partos vaginales. Sin embargo, estas diferencias no fueron estadísticamente significativas: $X^{2}=0.08 p>0.05$ (fig. 4).

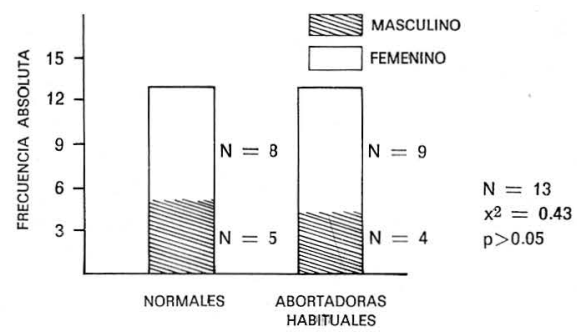

Fig. 3 - Sexo de los neonatos en los grupos de embarazadas normales y de pacientes con antecedentes de aborto habitual. No existe diferencia significativa.

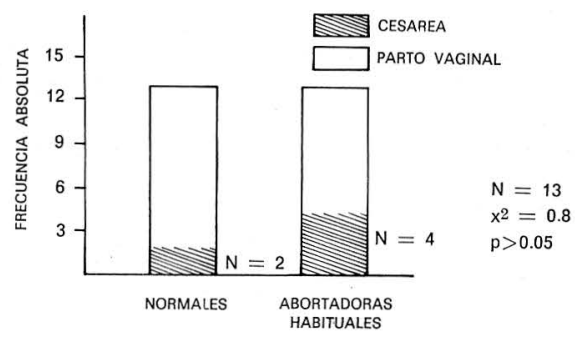

Fig. 4 - Proporción de partos terminados por via vaginal o por cesárea en los grupos de embarazadas normales y de embarazadas con antecedente de aborto habitual. No existe di. ferencia estadisticamente significativa entre ambos grupos. Creemos se debe al tamaño de la muestra.

\section{Puntaje de Apgar}

El puntaje de Apgar de los neonatos del grupo E. N. no fue diferente del grupo de A. H., ya que el promedio en ambas categorías de pacientes estudiadas fue exactamente igual: Apgar de 9 al $5^{\circ}$ minuto de vida. 


\section{Comentarios}

La primera evidencia experimental de que el cuerpo lúteo mantiene el embarazo surgió hacia el año de 1903 (10). A partir de esta fecha se han publica dos numerosos estudios que muestran una relación muy clara entre la progesterona, producida básicamente por el cuerpo lúteo y la fertilización, implantación del huevo y mantenimiento del embarazo $(11,12)$. Sin embargo, aunque han sido descritos por diversos autores ios diferentes niveles de progesterona durante la gestación en embarazos normales $(12,13)$, poco se conoce sobre la evolución de esta hormona en las pacientes con antecedentes de aborto habitual.

Los valores de progestinas encontrados por nosotros en embarazos normales son similares a los reportados previamente por otros investigadores $(9,13)$. Por el contrario, las concentraciones plasmáticas de progestinas en las pacientes abortadoras habituales, entre 5 y 9 semanas de gestación, se encuentran significativamente inferiores a las de las pacientes normales. En esta época, se inició la administración I. M. de gonadotropina coriónica y puede observarse cómo las concentraciones de progestinas plasmáticas en las abortadoras habituales empiezan a ascender. En las semanas 15 y 19 de la gestación, los valores de progestinas plasmáticas en el grupo de embarazadas normales y en las pacientes con aborto habitual son exactamente iguales. Se debe destacar que la única terapia que recibieron las pacientes $A$. $H$. fue la gonadotropina coriónica humana a la dosis ya mencionada.

Los anteriores resultados nos llevan a sugerir la administración de esta hormona en la prevención del aborto en la paciente A. H. como única terapia efectiva, hecho que se reporta por primera vez en la literatura.

Aunque la insuficiencia de progestinas, como se mencionó previamente, había sido implicada entre las etiologías más comunes del aborto habitual, los intentos terapéuticos para corregir esta deficiencia luteínica no habían sido exitosos $(3,4,5)$. Ya se mencionó, por ejemplo, cómo Shearman, Garret (5) encontraron que el caproato de 17 hidroxiprogesterona no incrementa los niveles de pregnandiol urinario cuando éste se encuentra normal o bajo, al comienzo de la gestación. Johansson (14) encontró que cuando administraba acetato de medroxiprogesterona a dosis altas después de la evaluación, los niveles plasmáticos de progesterona disminuían en vez de ascender. Cortés-Gallegos (15) ha reportado que los gestágenos sintéticos no solo no suplementan la hormona natural, sino que inclusive, tales agentes pueden ser luteolíticos.

Estudios anteriores de Csapo y Lipsett $(16,17)$ no encontraron actividad trofoblástica ni placentaria, a la 17 hidroxiprogesterona o a la 20 dihidroxiprogesterona, concluyendo que era necesaria la progesterona endógena para que esta actividad pudiera ocurrir. La anterior afirmación refuerza aún más nuestros comentarios en el sentido de tratar la deficiencia de progesterona provocando su liberación endógena por medio de la administración repetida de gonadotropina coriónica.

Por otra parte, los demás parámetros analizados, con excepción del peso al nacer, no mostraron diferencias significativas. La mayor proporción de parto operatorio (cesáreas) en el grupo de recién nacidos hijos de madres abortadoras habituales, podría explicarse por el hecho de que estos neonatos eran de mayor peso, además de ser productos de embarazos de alto riesgo. Sin embargo, las diferencias no fueron estadísticamente significativas. El sexo de los neonatos también fue sensiblemente igual en ambos grupos, así como el puntaje de Apgar al $5^{\circ}$ minuto de vida.

Finalmente, se debe destacar que en toda paciente abortadora habitual, antes 
de instituir el tratamiento con gonadotropina coriónica humana, es necesario destacar una etiología orgánica o cromosómica como causa del fracaso en las gestaciones previas. Una vez embarazada, se hace determinación basal de progestinas y precozmente se inicia el tratamiento con el fármaco mencionado a las dosis de 5.000 U. I. por V. I. M. cada tercer día hasta el $7^{\circ}$ mes de la gestación. A partir de esta época se suspende la administración de la gonadotropina coriónica y se deja evolucionar espontáneamente el embarazo. Queremos además recordar que, por tratarse de embarazos de alto riesgo perinatal, se debe vigilar continuamente el feto en el último trimestre de la gestación bien sea por métodos bioquímicos (particularmente el estriol plasmático) o biofísicos (análisis periódico de la frecuencia cardíaca fetal).

\section{RESUMEN}

El aborto espontáneo ocupa el primer lugar entre las causas de fracaso de la gestación. Dentro de este grupo de mujeres se encuentran las pacientes abortadoras habituales por deficiencia del cuerpo lúteo, para quienes se carecía de una terapia efectiva hasta la fecha. Intentos previos para corregir la deficiencia endógena de progesterona administrando por vía exógena esta hormona, no habían sido exitosos.

En el presente trabajo se intenta corregir esa deficiencia por medio de gonadotropina coriónica I. M., la cual provocará la liberación endógena de progesterona.

Es así como se seleccionaron dos grupos: uno constituido por 13 embarazadas normales y el segundo por $13 \mathrm{em}$ barazadas con antecedente de 3 o más abortos espontáneos y sin patología detectable. Las pacientes con aborto habitual tuvieron niveles bajos de progestinas plasmáticas al compararlos con las embarazadas normales. Sin embargo, después de administrar gonadotropina coriónica humana a las pacientes abortadoras habituales, los niveles de progestinas se hicieron sensiblemente iguales en ambos grupos y las 26 pacientes finalizaron exitosamente su gestación.

Sobre la base de los anteriores resultados se sugiere la administración periódica de cantidades conocidas de gonadotropina coriónica humana en la prevención del aborto en pacientes abortadoras habituales, por deficiencia de progesterona.

\section{HABITUAL ABORTION}

\section{SUMMARY}

Spontaneous abortion is in first place among the causes for gestation failure. Habitual abortive patients due to deficiency of the corpus luteum, for whom no effective therapy existed up to now, can be included in this group of women. All previous efforts to correct progesterone endo-deficiency administering this hormone in an exogenous way, have failed.

The purpose of this work is to correct this deficiency by means of I. M. chorionic gonadotropin, which will produce the endogenous liberation of progesterone.

In this way, two groups were selected: One was formed by 13 normal pregnant women and the other by 13 pregnant women with a background of 3 or more spontaneous abortions and with no detectable pathology. The patients with habitual abortion recorded low levels of plasmatic progestins when compared to normal pregnant women. Nevertheless, after administering human chorionic gonadotropin to habitual abortive patients, the progestin levels were almost equal in both groups and the 26 patients completed their gestation with success. 
Based of the above results, it is convenient to periodically administer known quantities of human chorionic gonadotropin in order to prevent abortion in habitual abortive women due to lack of progesterone.

Laboratory of Reproduction. Ginecology and Obstetrics Department. Valle University, Cali.

\section{BIBLIOGRAFIA}

1. WELTERDAL, P.: The frequency os espontaneous abortion. Acta obstet. ginec. scand. 25: 399, 1945.

2. HERTIG, A. T., ROCK, J., ADAMS, E. C., MENKIN, M. F.: A study of biologic wastage in early human pregnancy. Pediatrics 23: 202, 1959.

3. MALPAS, P.: A study of abortion sequences. J. Obstet. Gynaocol. Br. Emp. 45: 932, 1938.

4. POLAND, B. J., MILLER, J. R., Jones, D. C., TRIMBLE, B. K.: Reproductive couseling in patients who have had a spontaneous abortion. Am. J. Obstet. Gynecol. 127: 685, 1977.

5. SHERMAN, R. P., GARRET, W. J.: Doubie blind study of effect of 17-hydroxyprogesterone caproate on abortion rate. Br. Med. J. 1: 292, 1963.

6. GOLDZIEHER, J. W.: Double blind trial for a progestin in habitual abortion J. A. M. A. 188: 651, 1964.

7. KLOPPER, A., MACNAUGHTON, M. C.: Hormones in recurrent abortion. J. Obstet. Gynaecol. Br. Commonw. 72: 1022, 1965.
8. STROTT, C. A., YOSHIMI, T., ROSS, G. T., LIPETT, M. B.: Ovarios plupiology: -relation ship between plasma $\mathrm{LH}$ and steroidogenesis by the follide and corpus luteum; effect of HCG. J. Clin. Endocr. 29: 1157, 1969.

9. JOHANSSON, E. D. B.: The progesterone pattern of early pregnancy. Acta Endocrinol. 61: 607, 1969.

10. FRENKEL, L.: Die funtion des copues luteum. Arch. Gynaecol. 68: 438, 1903.

11. Corner, G. W., Allen, W. N.: Physiology of the corpus luteum. II. Production of a special uterine reaction (progesta ional proliferation) by extracts of the corpus luteum. Am. J. Physiol. 88: 326, 1929.

12. TULCHINSKY, D., OKADE, D. M.: Hormones in human pregnancy. IV. Plasma progesterone. Am. J. Obstet. Gynecol. 121: 293, 1975.

13. YANNONE, M. E., McCURDY, J. R., GOLDFINS, A.: Plasma progesterone leveis in normal pregnancy, labor and the puerperium. Am. J. Obstet. Gynecol. 101: 1058, 1968.

14. JOHANSSON, E. D. B.: Depression of the progesterone levels in women treated with synthetic gestagens after ovulation. Acta Endocrinol. 68: 779, 1971.

15. CORTES-GAllegos, V.: Agentes luteolíticos y reproducción. Gac. Med. Mex. 106: 259, 1973.

16. CSAPO, A. I., PULKKIMEN, M. O., TUTTNER, B.: The significance of the human corpus luteum in pregnancy maintenance. I Preliminary studies. Am. J. Obstet. Gynecol. 112: 1061, 1972.

17. LIPSETT, M. B., Cargille, C. M., ROSE, G. T.: Reproductive endocrinology. Methodologic advances and clinical studies. Ann. Intern. Med. 72: 933, 1970. 